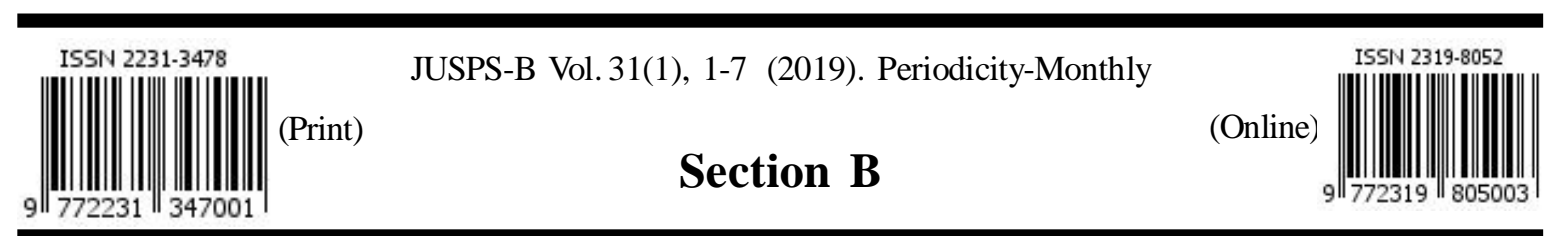

JOURNAL OF ULTRA SCIENTIST OF PHYSICAL SCIENCES
An International Open Free Access Peer Reviewed Research Journal of Physical Sciences
website:- www.ultrascientist.org

\title{
Demolition of Higgs mechanism
}

AMRIT SRECKO SORLI $^{1}$, DAVIDE FISCALETTI ${ }^{2}$ and SANTANU KUMAR PATRO ${ }^{3}$

\author{
${ }^{1}$ Foundations of Physics Institute, Slovenia \\ ${ }^{2}$ SpaceLife Institute, Italy \\ ${ }^{3}$ Department of Mathematics, Berhampur University, Odisha, India \\ Email Address for corresponding author: www.bijectivephysics.com, ksantanupatro@ gmail.com \\ http://dx.doi.org/10.22147/jusps-B/310101
}

Acceptance Date 30th January，2019， Online Publication Date 31st January, 2019

\begin{abstract}
A given model of the physical world is meant to be "scientific" if it is falsifiable. Falsifiable model is not irrefutable truth; there is a possibility to be proven wrong. This gives science a chance of improvement. Higgs mechanism is not falsifiable and cannot be considered "scientific"; rather it is "pseudo-science". Higgs boson is artificially made up momentary flux of energy, released here and there, in proton collisions and does not prove the existence of the Higgs field.

Key words: Higgs mechanism, standard model, falsifiability, bijective analysis.
\end{abstract}

\section{Introduction}

Karl Popper said: "A theory should be considered scientific if, and only if, it is falsifiable"1. A scientific statement is one that could possibly be proven wrong. Such a statement is said to be falsifiable. The falsifiable statement always remains tentative and open to the possibility that it is wrong.

We can read following regarding the Higgs mechanism: "All of space is assumed to be filled with a Higgs field - a background sea of virtual Higgs bosons that pop in and out of existence. The quarks, leptons, and $W$ and $Z$ bosons moving around through space interact with this field, which is why these particles have mass. The photons and gluons do not interact with the Higgs field, which is why these particles do not have mass. Even the Higgs boson itself interacts with the Higgs field. It gives itself mass!"2.

This statement is not falsifiable and cannot be considered scientific. Especially the last sentence is a

This is an open access article under the CC BY-NC-SA license (https://creativecommons.org/licenses/by-nc-sa/4.0) 
classic example of a statement which is not falsifiable: "It (Higgs boson) gives itself mass !" This is very similar to the statement: "God has made himself !"

Bijective analysis of Standard Model :

Regarding Higgs mechanism the Standard model says: "Particles exist in an empty space. All particles are mass-less. Some field must exist, which gives mass to the particles". This view has historical background, namely, with the abolishing of ether Einstein has introduced the idea of "empty space".

Bijective analysis of Standard Model shows that the idea of "empty space" has no bijective correspondence in the physical world. We all agree in physics that particles which exist in space are "energy" which means that space is also energy. Energy is "physical" and space is "physical" too. Quantum vacuum is the physical origin of space. Elementary particles are different forms of space energy, see figure 1 below:

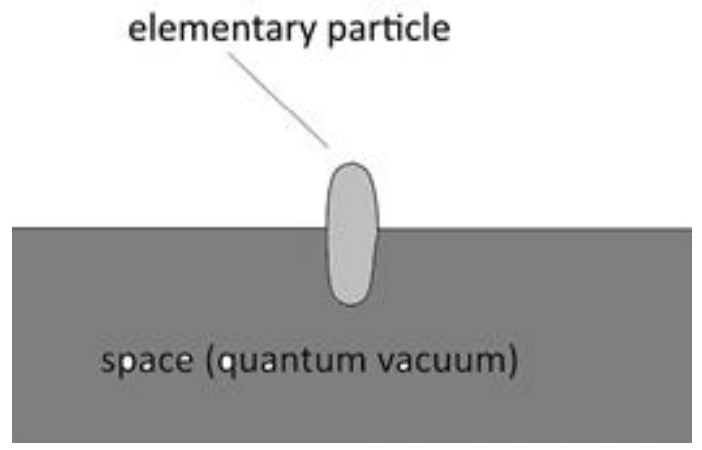

Figure 1: Elementary particle is the structure of space energy

The idea that all particles are mass-less is not in accord with the "mass-energy equivalence principle". Photons and gluons also have mass according to the following formula:

$$
m=\frac{h \cdot v}{c^{2}}
$$

where $h$ is Planck constant and $v$ is frequency.

Photons and gluons have a mass as the amount of their energy. They do not have rest mass and they do not have inertial mass. Rest mass of the proton is its mass when it is still regarding the space in which it exists. Advanced Relativity is reintroducing space as the absolute reference system. Proton rest mass is diminishing energy density of space in the centre of the proton according to the following equation:

$$
\rho_{S E}=\rho_{P E}-\frac{m \cdot c^{2}}{V}
$$

where $\rho_{S E}$ is energy density of space in the centre of the proton, $\rho_{P E}$ is Planck energy density characteristic for the interstellar space, $V$ is the volume of the proton.

Higher energy density of outer space is pushing towards lower energy density in the centre of the proton which generates proton's inertial mass, see figure 2 below: 


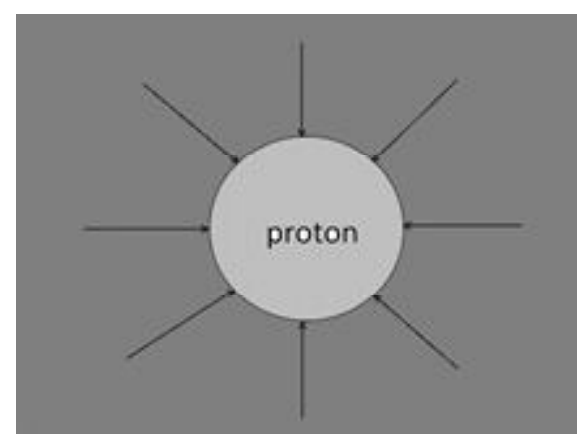

Figure 2: Proton inertial mass

When neutron is close to the proton (in atom nucleus) they both create the area of lower energy density of space which generates strong nuclear force between them, see figure 3 below:

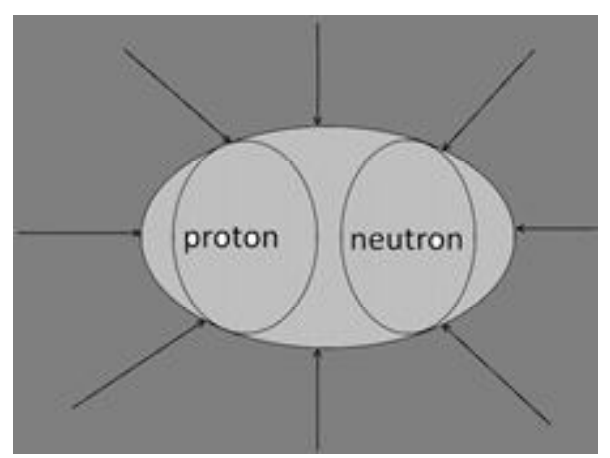

Figure 3: Strong nuclear force between proton and neutron in deuterium atom

Also two atoms are diminishing energy density of space which generates gravity. Gravity force is the pushing force of the higher energy density of the space towards lower energy density of the space, see figure 4 below:

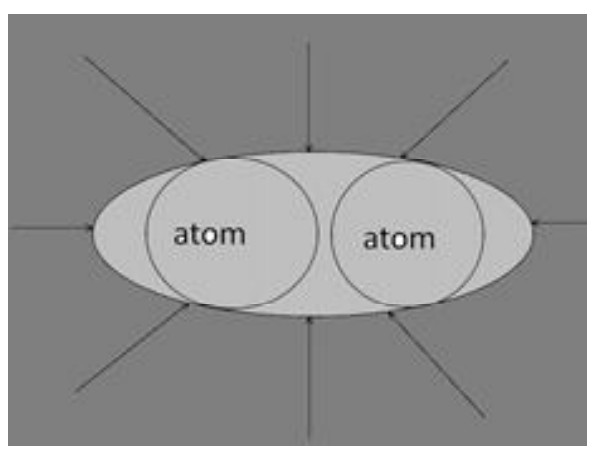

Figure 4: Gravity between two atoms

Gravity force and strong nuclear force have the same origin which is the diminished energy density of space in the centre of massive particles, physical objects and stellar objects.

When proton is accelerated in LHC it interacts with the energy of the space. We call this effect 
"dragging effect".

Higher is the velocity of the proton (in regard to the space) bigger is its relativistic energy which actually is its kinetic energy:

$$
E=\gamma \cdot m \cdot c^{2}
$$

where $\gamma$ is Lorentz factor and $m$ is the rest mass of the proton. formula:

Energy density of space in the centre of relativistic particle is diminishing according to the following

$$
\rho_{S E}=\rho_{P E}-\frac{\gamma \cdot m \cdot c^{2}}{V}
$$

Combining formula (3) and (4) we get formula:

$$
E=\gamma \cdot m \cdot c^{2}=\gamma \cdot\left(\rho_{P E}-\rho_{S E}\right) \cdot V
$$

where $E$ is the relativistic energy of the proton. Formula (5) shows that relativistic energy of the proton has its source in the energy of space which is absorbed in the proton because of the dragging effect. The right side of the equation is showing from where the energy of relativistic proton came from.

When two relativistic protons collide, the proton falls apart in several sparkles of energy which soon disappear back into the primordial energy of the space. Higgs boson is one of these sparkles. Higgs boson is artificially made particle which has no existence in the physical universe and does not prove the existence of Higgs field.

\section{Bijective analysis of the proton:}

Michael Friedlander proposed that protons have the origin in the intergalactic space ${ }^{5}$. Today's astronomy is not taking his consideration as the plausible one, because of the prevalent belief that universal space is empty. According to bijective analysis, universal space is the primordial energy of the universe. In interstellar areas energy density of space is at the maximum. Because of this physical circumstance energy of space is getting formed in the protons. In black holes energy density of the space is at the minimum. Because of this circumstance proton becomes unstable and atoms disintegrate back into the primordial energy of the space. This circulation of the energy has no beginning and no end. The universe is a non-created system in a permanent dynamic equilibrium ${ }^{3}$.

According to the bijective analysis, proton has no constitutive parts. Proton is the vortex of space energy.

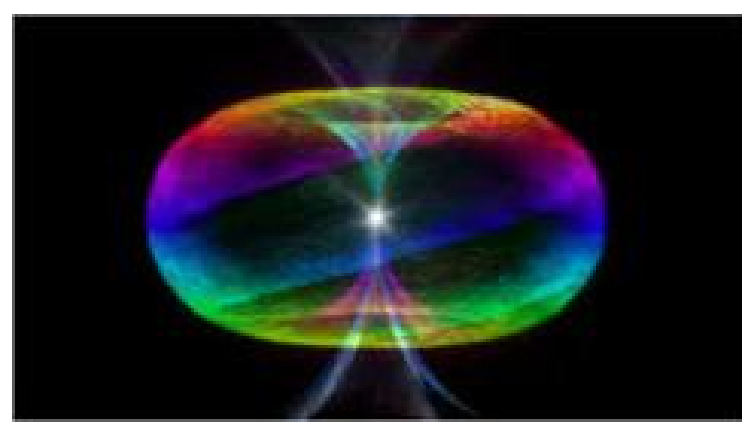

Figure 5: Proton as the vortex of space energy 
Standard model imagination that proton is made out of quarks is not real. Quarks are belonging to the same group of the particles as the Higgs boson. The group is named "Artificial Particles" (AP). Artificial particles are momentary fluxes of energy released in cyclotrons collisions. Research of the particles in AP group will never give technological application and represents an enormous waist of financial resources with no return. Giving this money in past 30 years for quantum vacuum research we would have already technologies for the extraction of energy from the space and we would develop antigravity.

Proton, electron and photon, belong to the "Constitutive Particles" (CP). Constitutive particles are basic elements of more complex systems (atoms) which exist in physical reality. The main criterion for the particles belonging to the AP group or to the CP group is their lifetime. Neutron for example is not belonging to the $\mathrm{CP}$ group. Neutron is a complex system constituted by one proton and one electron.

The life time of Muon is $2,2 \cdot 10^{-6} \mathrm{~s}$. The Muons arriving on the Earth's surface are created indirectly as decay products of collisions of cosmic rays with particles of the Earth's atmosphere. They can also be artificially created in cyclotrons. Thinking that muons are particles in the sense that they are constitutive elements of some more complex systems, is absolutely wrong. In this sense mouns belong to the third group of particles named "Transitory Particles" (TP). Transitory particles have no actual physical existence; they are only the segment of a given physical process. That's why they have extremely short life time.

The life time of 'Tau lepton' is $2,9 \cdot 10^{-13} s$. It belongs to AP group. Neutral pion life time is $8,4 \cdot 10^{-17} s$. It belongs to AP group. Charged pion life time is $2,6 \cdot 10^{-8} s$. It belongs to AP group. W boson and $\mathrm{Z}$ boson life times are $10^{-25} \mathrm{~s}$. W boson and $\mathrm{Z}$ boson belong to TP group. They are the result of the atom nucleus instability which causes radioactive decay ${ }^{6}$.

Bijective physics (BP) classifies elementary particles into three groups:

\section{BIJECTIVE PHYSICS ELEMENTARY PARTICLES SCHEME}

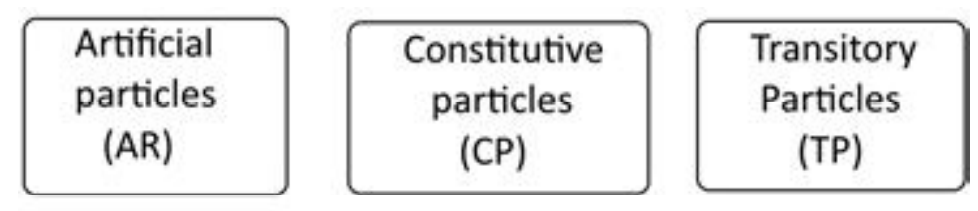

\section{All elementary particles are different structures of space (quantum vacuum).}

Figure 6: Bijective Physics elementary particles scheme

In Bijective physics model, 'dark energy' is the energy of space itself. Five percent of energy is in the dorm of atoms (matter), and ninety five percent of energy is in the form of space. Energy in space is non-created, primordial. Energy of matter is structured energy of space. Energy of space obeys the 'syntropy law' and matter obeys the "entropy law' ${ }^{6}$. Constitutive particles are stable forms of space energy and that is why it has almost "infinite lifetime". Bijective analysis confirms that any scientific research on (AR) group and (TP) group, will 
never give technological implementation. Rather, Research on physical properties of space will give free energy technology and antigravity technology.

Local space and general space :

Each elementary particle is the structure of the space energy and has its own local space. This local space with the diminished energy density, which corresponds to the energy of the particle, cannot be seen as something separate from the particle. Standard model sees particles are separated from the space. Bijective analysis confirms that the particle and its local space are one entity. This is valid also for both the massive objects and stellar objects. They all have their own local space. Interaction between local space and the general space has the outcome as 'inertial mass' and 'gravitational mass'. When the object is moving in the space, the interaction between local space and general space results in the 'dragging effect'.

Local space of the planet Earth is moving and rotating with the Earth. Rotation of local space of the Earth explains the 'Sagnac effect', which can't be explained through Special Theory of Relativity. The light needs more time when moving from the point $B$ to the point $A$ in the direction which is opposite to the Earth rotation. When light is moving from the $\mathrm{A}$ to $\mathrm{B}$, it is moving in rotating local space and so it needs less time ${ }^{7}$.

In bijective physics model a photon is the wave of space. Local space is moving with the Earth; Earth is not moving in some stationary ether as predicted by physics of $19^{\text {th }}$ century. Michelson-Morley experiment was designed on the wrong preposition that Earth is moving in some stationary ether. The light, which is entering the interferometer is the wave of the local space, that's why Michelson-Morley experiment has given null result. Negative outcome of Michelson-Morley has lead to the false idea, namely, that photon is moving in an "empty space". This interpretation of Michelson-Morley experiment does not resurrect the ether model. Bijective analysis confirms that there is no "empty space" and there is no "ether". Space is the only primordial energy of the universe and light is the wave of space ${ }^{7}$.

Standard model states that particles and massive bodies exist in empty space. This view has no more power to explain the origin of inertial mass and gravitational mass. But in accordance with 'bijective physics', particles and massive bodies are different structures of the space energy. Interaction between local space and general space gives origin to the inertial mass, gravitational mass and dragging effect.

\section{Conclusions}

It seems that 'Higgs mechanism' has become the religion of today's physics. Higgs boson, which is not more than the artificial manmade momentary flux of energy released here and there in the proton collisions, has become the "holy grail" of today's physics, so-called "God particle". The progress of our understanding of the origin of mass is based on the pillars as Einstein's "mass-energy equivalence", "inertial mass gravitational mass equivalence", "Karl Popper work", and "bijective research methodology".

\section{References}

1. Karl Popper, Critical Rationalism, https://www.iep.utm.edu/cr-ratio/ (2019).

2. The Physics Hypertext Book https://physics.info/standard/ (2019).

3. Sorli A., Dobnikar U., Patro S. K., Mageshwaran M. and Fiscaletti D., "Euclidean-Planck Metrics of Space, Particle Physics and Cosmology", NeuroQuantology 16, 4, 18-25 (2018). 
https://www.neuroquantology.com/index.php/journal/article/view/1221/961

4. Amrit Srecko Sorli, Higgs boson Demolition, Amazon (2019).

5. Friedlander MW. A thin cosmic rain: Particles from outer space, Harvard: Harvard University Press, 2002.

6. Sorli A., Patro S.K, Fiscaletti D., NeuroQuantology 16(11), 55-67 (2018).

Unified Field Theory Based on Bijective Methodology

https://www.neuroquantology.com/index.php/journal/article/view/1855/1287

7. Fiscaletti D., Sorli A., Dynamic Quantum Vacuum and Relativity, Annales Physica, Vol. 71 (2016).

http://journals.umcs.pl/aaa/article/view/2808 